International Journal of Pure and Applied Mathematics

Volume 89 No. 3 2013, 357-364

ISSN: 1311-8080 (printed version); ISSN: 1314-3395 (on-line version)

url: http://www.ijpam.eu

doi: http://dx.doi.org/10.12732/ijpam.v89i3.7

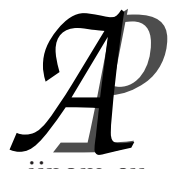

ijpam.eu

\title{
ON THE K-RING OF THE CLASSIFYING SPACE OF THE DIHEDRAL GROUP
}

\author{
Mehmet Kirdar \\ Department of Mathematics \\ Faculty of Arts and Sciences \\ Namik Kemal University \\ Tekirdag, TURKEY
}

\begin{abstract}
We describe the K-ring of the classifying space of the dihedral group in terms of generators and the minimal set of relations by emphasising the connection with the polynomials arising in the KO-rings of lens spaces and demonstrating the generators of the filtrations of the Atiyah-Hirzebruch spectral squence.
\end{abstract}

AMS Subject Classification: 55R50, 20C10

Key Words: topological K-theory, representation theory, dihedral groups

\section{Introduction}

Let $D_{2 n}$ denote dihedral group with $2 n$ elements. In this note, by collecting enough evidence from the the Atiyah-Hirzebruch Spectral Sequence (AHSS) and essentially by making use of the structure of the representation ring $R\left(D_{2 n}\right)$, we will describe $K\left(B D_{2 n}\right)$ in terms of generators and the minimal set of relations by means of the Atiyah-Segal Completion Theorem (ASCT).

The $K$-rings of the $B D_{2 n}$ are studied classically in [3]. For the computations in the extended cohomology theory and its connective version, see [1]. We note that the zeroth part of the $K$-cohomology and hence the relations here should be extracted from the results in [1]. But, they are not explicitly written there.

Received: July 18, 2013

(c) 2013 Academic Publications, Ltd. url: www.acadpubl.eu 
First we deal with the case where $n$ is odd and we will have a complete description.

Then, we will consider the even case which turns out to be quite complicated compared to the odd case. Thanks to [4], we could be able to find the minimal relations in that case quickly. We also explain the twisted nature of the AHSS by demonstrating the generators of its filtrations.

\section{The Odd Case}

Let $n=2 k+1$ and $k \geq 1$. Isomorphism classes of the irreducible complex representations of $D_{2 n}$ are the two one dimensional representations $\eta, 1$ and the $k$ two dimensional representations $\rho_{i}$ where $1 \leq i \leq k$. Actually, assuming that $\rho_{0}=1+\eta$, for any $i \in Z$ the symbol $\rho_{i}$ makes sense and we have the relations $\eta^{2}=1, \rho_{i}=\eta \rho_{i}=\rho_{n-i}=\rho_{n+i}$ and $\rho_{i} \rho_{j}=\rho_{i+j}+\rho_{j-i}$ for all $i, j \in Z$ in the representation ring $R\left(D_{2 n}\right)$.

Let us denote the complex vector bundles induced from these representations over $B D_{2 n}$ by the same letters.

The integral group cohomology of $D_{2 n}$, for $n$ odd, is given in [2] as

$$
H^{p}\left(B D_{2 n} ; Z\right)= \begin{cases}Z & p=0 \\ Z_{2} & \text { if } p=4 s+2 \\ Z_{n} \oplus Z_{2} & \text { if } p=4 s, s \geq 1 \\ 0 & \text { if } p \text { is odd. }\end{cases}
$$

and since the cohomology in odd dimensions is zero, AHSS collapses on the second page and we can read the filtrations of the ring $K(B G)$ from the cohomology.

We define the reductions $v=\eta-1$ and $\phi=\rho_{1}-2$ in the ring $K\left(B D_{2 n}\right)$. Since the representations $\eta$ and $\rho_{1}$ generate the representation ring $R\left(D_{2 n}\right)$, $v$ and $\phi$ generate $K\left(B D_{2 n}\right)$ due to ASCT. The relation $\eta^{2}=1$ in our new variables is

$$
v^{2}=-2 v .
$$

From $\rho_{1}=\eta \rho_{1}$ we get $(\phi+2) v=0$ or equivalently

$$
\phi v=v^{2} .
$$

The classifying space $B Z_{2}$ is the infinite dimensional real projective space and its $K$-ring is generated by $v$ modulo the relation $v^{2}=-2 v$ where $v=\eta-1$ is the reduction of the tautological complex line bundle $\eta$ over $B Z_{2}$. 
We have two natural group injections of $Z_{2}$ in the group $D_{2 n}$. Let us pick one of them $Z_{2}<D_{2 n}$. This induces a ring homomorphism $i: K\left(B D_{n}\right) \rightarrow K\left(B Z_{2}\right)$. Now we should have $i(v)=v$ by naturality of the constructions. Since $v^{s}$, $s \geq 1$, is generating $E_{\infty}^{2 s,-2 s}$ filtration of $K\left(B Z_{2}\right)$ in AHSS, the same is true for $K\left(B D_{n}\right)$ by naturality and this explains the $Z_{2}$ parts of the cohomology and the filtrations $E_{\infty}^{2 s,-2 s}=E_{2}^{2 s,-2 s}$ of the AHSS. Now we should explain the remaining $Z_{n}$ parts on dimensions $4 s$.

The classifying space $B Z_{n}$, for $n \geq 3$, is an infinite dimensional lens space and its $K$-ring is generated by $\mu$ modulo the relation $(1+\mu)^{n}=1$ where $\mu=\eta-1$ is the reduction of the tautological complex line bundle $\eta$ over $B Z_{n}$. We have a natural group injection $Z_{n}<D_{2 n}$ which induces a homomorphism $j: K\left(B D_{n}\right) \rightarrow K\left(B Z_{n}\right)$, as explained above for the naturally identified $Z_{2}$ subgroups of the dihedral group. Then we have $j(v)=0$ and $j(\phi)=\eta+\eta^{-1}-2$ in $K\left(B Z_{n}\right)$.

Now, this last reduced bundle is the generator $c(w)=\eta+\eta^{-1}-2$ of the image of the $K O$-ring of $Z_{n}$ under the complexification $c: K O\left(B Z_{n}\right) \rightarrow K\left(B Z_{n}\right)$ which is injective. Here $w$ is the realification of $\mu$ and it generates $K O\left(B Z_{n}\right)$. Recall that when $n$ is odd

$$
K O\left(B Z_{n}\right)=Z[w] /\left(w f_{n}(w)\right)
$$

where

$$
w f_{n}(w)=\psi^{\frac{n+1}{2}}(w)-\psi^{\frac{n-1}{2}}(w)
$$

and where $\psi^{i}(\cdot)$ are the polynomials defined by $\psi^{i}(w)=\sum_{j=1}^{i} \frac{\left(\begin{array}{c}i \\ j\end{array}\right)\left(\begin{array}{c}i+j-1 \\ j\end{array}\right)}{\left(\begin{array}{c}2 j-1 \\ j\end{array}\right)} w^{j}$ for $i \geq$ 1. Note that this is how the Adams operations are acting on $w$ and $K O\left(B Z_{n}\right)$ is just the fixed set of the complex conjugation.

The polynomial $f_{n}(w)$ is explicitly given by

$$
f_{n}(w)=n+\sum_{j=1}^{\frac{n-3}{2}} \frac{n\left(n^{2}-1^{2}\right)\left(n^{2}-3^{2}\right) \ldots\left(n^{2}-(2 j-1)^{2}\right)}{2^{2 j} \cdot(2 j+1) !} w^{j}+w^{\frac{n-1}{2}} .
$$

We will call $f_{n}(w)$ the minimal polynomial of the $\operatorname{ring} K O\left(B Z_{n}\right)$. If $n=p$ is an odd prime number then $f_{n}(\cdot)$ is the minimal polynomial of the number $2 \cos \left(\frac{2 \pi}{p}\right)-2$. This can be proved by using the Eisenstein's criterion and factorization of odd indexed Chebishev polynomials.

Now, since $E_{\infty}^{4 s,-4 s}$ filtrations of $K\left(B Z_{n}\right)$ and $K\left(B D_{n}\right)$ coincides on $Z_{n}$ parts by means of $j$, and since $\phi v=v^{2}=-2 v$, we deduce that $\phi$ satisfy a relation in the form $\phi f_{n}(\phi)+c v=0$ where $c$ is an integer to be determined. 
Multiplying this relation by $v$ and by using $\phi v=v^{2}$ we have $v^{2}\left(f_{n}(v)+c\right)=0$ and by using $v^{2}=-2 v$, we conclude that $c=-f_{n}(-2)$.

We did a little calculation by using the various relations, essentially the iterative relation $\rho_{i} \rho_{j}=\rho_{i+j}+\rho_{j-i}$ of the representation ring, with some help of the compute option of the Scientific WorkPlace. We observed that, indeed, for small values of $k, \phi$ satisfies a relation in the form $\phi f_{n}(\phi) \pm v=0$. Let's explain why $c$ is \pm 1 . It is known that $f_{n}(\phi)$ is the square root of the polynomial

$$
\frac{2 T_{n}\left(\frac{\phi+2}{2}\right)-2}{\phi}
$$

where $T_{n}(\cdot)$ is the Chebishev polynomial of degree $n$ and $n$ is odd. This last expression is just equal to 1 when we substitute $\phi=-2$, because $T_{n}(0)=0$. We conclude that $f_{n}(-2)= \pm 1$.

All these information fit together very nicely except the filtration absurdity in the main relation

$$
\phi f_{n}(\phi)=f_{n}(-2) v \text {. }
$$

On the left hand side we have something which seems to be of filtration at least four 4 while the right hand side is of filtration 2 . This may be modified by claiming that $Z_{n}$ part of the filtration $E_{\infty}^{4 s,-4 s}$ is generated by $\phi-v$. In fact, this argument seems to be justified in [3]. This kind of a generator in a filtration can be seen as some kind of twisting phenomenon in the $K$-cohomology. But in any case, we have the following

Theorem 1. Let $n \geq 3$ be odd. Then

$$
K\left(B D_{2 n}\right)=Z[v, \phi] /\left(v^{2}+2 v, v \phi+2 v, \phi f_{n}(\phi)-f_{n}(-2) v\right)
$$

where $f_{n}(\cdot)$ is the minimal polynomial of $K O\left(B Z_{n}\right)$; in particular the minimal polynomial of $2 \cos \frac{2 \pi}{n}-2$ when $n$ is prime.

Let us make some concluding remarks. It is interesting that the even part of the integral cohomology of the group, and thus the whole integral cohomology, is completely detected by and detects the $K$-ring when $n$ is odd. Since, the representations of the dihedral groups are real, the rings $K\left(B D_{2 n}\right)$ and $K O\left(B D_{2 n}\right)$ should be isomorphic as rings, but, geometrically they carry different information. Their spectral sequences are different and $K O\left(B D_{2 n}\right)$ should be related to some of the cohomology with $Z_{2}$ coefficients.

And note also that the curious constant in the theorem above seems to be

$$
f_{n}(-2)=\sin \frac{k \pi}{2}+\cos \frac{k \pi}{2}
$$


where $n=2 k+1$.

\section{The Even Case}

The even case is quite complicated with respect to the odd case. But, recently, Kirdar and Sevilay, [4], described the $K$-ring of the generalized quaternion groups by a minimal set of relations and we realized that the relations of the representation ring here are very similar to the relations there. And amazingly, we could be able to easily deduce the minimal set of relations for the dihedral groups, the even case, with some little modifications.

Since $D_{4}$ is $Z_{2} \times Z_{2}$, the space $B D_{4}$ is the product of two infinite dimensional real projective spaces, and the $K$-ring can be described by Atiyah's Kunneth Theorem for $K$-cohomology.

So, let us take $n=2 k$ and $k \geq 2$ from now on. The representation ring $R\left(D_{4 k}\right)$ is generated by the four one dimensional representations $1, \eta_{1}, \eta_{2}$ and $\eta_{3}=\eta_{1} \eta_{2}$ and the $k-1$ two dimensional representations $\rho_{i}$ where $1 \leq i \leq k-1$. Actually, $\rho_{i}$ make sense for any integer $i$, due to the standard definition of these representations by matrices.

The various relations in $R\left(D_{4 k}\right)$ are: The start $\rho_{0}=1+\eta_{3}$ and the end $\rho_{k}=\eta_{1}+\eta_{2} ; \eta_{1}^{2}=\eta_{2}^{2}=\eta_{3}^{2}=1 ; \eta_{r} \rho_{i}=\rho_{k-i}=\rho_{k+i}$ for $r=1,2$ and $\eta \rho_{i}=\rho_{i}$; $\rho_{n+i}=\rho_{i}$; and the main relation $\rho_{i} \rho_{j}=\rho_{i+j} \rho_{i-j}$ for all $i, j \in Z$.

The representations above induce vector bundles over the space $B D_{4 k}$. We will denote the induced bundles over $B D_{4 k}$ again by the same letters and set the reductions $v_{1}=\eta_{1}-1, v_{2}=\eta_{2}-1, v_{3}=\eta_{3}-1$ and $\phi=\rho_{1}-2$ in $K\left(B D_{4 k}\right)$.

As we said before, the results of [4] can be copied here with some modifications. The relations above are exactly the same relations in [4] written for the generalized quaternion group $Q_{4 k}$, except that the start relation and the end relation are interchanged! Because of that, it is meaningful to choose the pair of representations $\eta_{2}, \eta_{3}$ instead of the pair $\eta_{1}, \eta_{2}$ in our problem as the generators in the description. Note that, since $\eta_{3}=\eta_{1} \eta_{2}$, we have $v_{3}=v_{1}+v_{2}+v_{1} v_{2}$ and although highly convoluted, we can write the description by using $v_{1}$ and $v_{2}$, whenever we want.

Now, since $\eta_{2}, \eta_{3}$ and $\rho_{1}$ generate the representation ring, $v_{2}, v_{3}$ and $\phi$ generate $K\left(B D_{4 k}\right)$ due to ASCT. Now, we can transform the relations in the representation ring to our new variables and we can write the following:

Theorem 2. Let $n=2 k$ and $k \geq 2$. Then $K\left(B D_{2 n}\right)$ is generated by $v_{2}, v_{3}$ and $\phi$ and the minimal set of relations 


$$
\begin{aligned}
& v_{2}^{2}=-2 v_{2} \\
& v_{3}^{2}=-2 v_{3} \\
& v_{2} \phi=\psi^{k-1}(\phi)-\phi-2 v_{2}-v_{3} \\
& v_{2} \phi=\psi^{k-1}(\phi)-\phi-2 v_{2} \\
& v_{3} \phi=-2 v_{3} \\
& v_{2} v_{3}=4 \phi+\phi^{2}-2 v_{2}-2 v_{3} \\
& v_{2} v_{3}=\psi^{k}(\phi)-2 v_{2}-v_{3} \\
& v_{2} v_{3}=\psi^{k}(\phi)-2 v_{2}
\end{aligned}
$$

(3, for $k \geq 3$ and $k$ is odd)

(3, for $k \geq 3$ and $k$ is even)

$(5$, for $k=2)$

(5, for $k \geq 3$ and $k$ is odd)

(5, for $k \geq 3$ and $k$ is even)

Proof. Thanks to [4], we derived these relations quickly from the Theorem 1, [4]. But, there is a slight modification for the case $k$ is odd in the relations 3 and 5 above. Now, let us analyze this quick and surprising result through the AHSS, confirming the minimality of the relations and thus completing the proof of the theorem. Before that, let us recall the cohomology.

The integral cohomology of the group $D_{2 n}$ is given by, [2],

$$
H^{p}\left(B D_{2 n} ; Z\right)= \begin{cases}Z & p=0 \\ Z_{n} \oplus\left(Z_{2}\right)^{2 s} & \text { if } p=4 s, s \geq 1 \\ \left(Z_{2}\right)^{2 s} & \text { if } p=4 s+1 \\ \left(Z_{2}\right)^{2 s+2} & \text { if } p=4 s+2 \\ \left(Z_{2}\right)^{2 s+1} & \text { if } p=4 s+3\end{cases}
$$

and we can read the filtrations of the second page of the AHSS from the cohomology described above. But, since, the odd dimensional cohomology is not trivial, AHSS may not collapse on page 2. And in fact, this is the case here.

It is enough to consider $k=2$ in order to explain the minimality of the relations through the AHSS, since exactly the same explanation goes for $k \geq 3$.

Now, we have two natural group inclusions $Z_{2}<D_{8}$ and they induce two natural ring homomorphisms $j_{1}: K\left(B D_{8}\right) \rightarrow K\left(B Z_{2}\right)$ and $j_{2}: K\left(B D_{8}\right) \rightarrow$ $K\left(B Z_{2}\right)$. Recall that $K\left(B Z_{2}\right)=Z[v] /\left(v^{2}+2 v\right)$. Now, since under $j_{1}$ and $j_{2}, v_{1}$ and $v_{2}$ maps to $v$, we deduce that $E_{\infty}^{2,-2}=Z_{2} \oplus Z_{2}$ of the AHSS of $K\left(B D_{8}\right)$ is generated by $v_{1}$ and $v_{2}$. Note that $v_{1}=v_{2}+v_{3}+v_{2} v_{3}$ here.

For $K\left(B D_{8}\right)$, the main relation is $4 \phi=-v_{2}^{2}-v_{3}^{2}+v_{2} v_{3}-\phi^{2}$. From this, we have $4 \phi=$ h.o.t. and we claim that the $Z_{4}$ part of the filtration $E_{\infty}^{4,-4}=Z_{4} \oplus$ $Z_{2} \oplus Z_{2}$ is generated by $\phi$. Really, we have a natural group inclusion $Z_{4}<D_{8}$, and this induces a natural ring homomorphisms $j: K\left(B D_{8}\right) \rightarrow K\left(B Z_{4}\right)$. Recall that $K\left(B Z_{4}\right)=Z[\mu] /\left((1+\mu)^{4}-1\right)$. Under $j, \phi$ maps to the infinite alternating sum $\mu^{2}-\mu^{3}+\mu^{4}-\ldots$ and since $\mu^{2}$ generates $E_{\infty}^{4,-4}$ filtration of $K\left(B Z_{4}\right)$, by naturailty, $\phi$ should generate a direct summand of $E_{\infty}^{4,-4}$ for $K\left(B D_{8}\right)$ too. And there is one choice. 
We claim that $Z_{2} \oplus Z_{2}$ part of $E_{\infty}^{4,-4}$ is also surviving and is generated by $v_{1} v_{3}$ and $v_{2} v_{3}$ respectively. First of all, $j_{1}$ and $j_{2}$ should be non-trivial on these filtrations, because $E_{\infty}^{4,-4}$ of $K\left(B Z_{2}\right)$ 's are generated by $v^{2}$. Since, under $j_{1}$ and $j_{2}, \phi$ which generates $Z_{4}$ part of the filtration $E_{\infty}^{4,-4}$, maps to zero, only the other parts can provide this non-triviality. On the other hand, under $j_{1}$ and $j_{2}$, the element $v_{3}$ goes to $v$ so that $v_{1} v_{3}$ and $v_{2} v_{3}$ both go to $v^{2}$. And the claim is proved. Note that, $Z_{2} \oplus Z_{2}$ part of $E_{\infty}^{4,-4}$ can not be generated by $v_{1}^{2}$ and/or $v_{2}^{2}$. This is very confusing, but, under $j$, since both $v_{1}$ and $v_{2}$ go to $2 \mu+\mu^{2}, v_{1}^{2}$ and $v_{2}^{2}$ go to $-2 \mu^{3}-3 \mu^{4}-\mu^{5}$, and they are related to the filtration $E_{\infty}^{6,-6}$.

So far, we noticed that the AHSS is collapsing on the second page for the filtrations $E_{\infty}^{2,-2}$ and $E_{\infty}^{4,-4}$. But, this will not be the case for the higher filtrations.

We claim that $E_{\infty}^{6,-6}$ is $Z_{2} \oplus Z_{2} \oplus Z_{2}$ and that it is generated by $v_{1} v_{3}^{2}$, $v_{2} v_{3}^{2}$ and $v_{1} v_{2}$. Note that on the second page, $E_{2}^{6,-6}$ is $Z_{2} \oplus Z_{2} \oplus Z_{2} \oplus Z_{2}$ and it has four summands. We claim that one summand $Z_{2}$ can not survive to infinity. This claim is not difficult to prove after the observations we did on the filtrations $E_{\infty}^{2,-2}$ and $E_{\infty}^{4,-4}$. First of all, $v_{1} v_{3}^{2}, v_{2} v_{3}^{2}$ should survive on the filtration $E_{\infty}^{6,-6}$, because they go to $v^{2}$ under $j_{1}$ and $j_{2}$. This takes care of two summands. The product $v_{1} v_{2}$ has clearly on the filtration $E_{\infty}^{6,-6}$ because under $j$ it goes to where $v_{2}^{2}$ goes, due to $v_{2}^{2}=v_{1} v_{2}+v_{2} v_{3}$. And this takes care of the third summand. Because of the relations, we exhausted all elements that can live on the filtration $E_{\infty}^{6,-6}$ and we are done.

Now, the picture is clear for $K\left(B D_{8}\right)$ for the higher filtrations: Firstly, the filtration $E_{\infty}^{4 t,-4 t}$ is $Z_{4} \oplus Z_{2} \oplus Z_{2}$ and it is generated by $\phi$ and $v_{1} v_{3}^{2 t-1}, v_{2} v_{3}^{2 t-1}$ respectively, where $t \geq 1$. Secondly, the filtration $E_{\infty}^{4 t+2,-4 t-2}$ is $Z_{2} \oplus Z_{2} \oplus Z_{2}$ and it is generated by $v_{1} v_{3}^{2 t}, v_{2} v_{3}^{2 t}$ and $v_{1} v_{2}^{t}$ respectively, where $t \geq 1$.

Finally, $2 v_{2}$ can be expressed by $\phi, v_{2} v_{3}$ for the even case and by $\phi, v_{2} v_{3}$ and $v_{3}$ for the odd case by means of the relation 5 . Since $v_{3}=v_{1}+v_{2}+v_{1} v_{2}$, we deduce that $2 v_{2}$ can be expressible by the elements we chose as the generators of the filtrations of the AHSS.

We also note that the generator $\phi$ satisfies the relation

$$
g_{2 k}(\phi)=\psi^{k+1}(\phi)-\psi^{k-1}(\phi)=0 .
$$

The polynomial $g_{2 k}(\phi)$ is explicitly given in [4]. It should be clear to the reader that this polynomial is the even version of the magical polynomial $w f_{n}(w)$ of the odd case.

As a final note, we point out the very natural continuation of the problem: $K$-rings of the classifying spaces of the symmetric groups. For $n=3, D_{6}=S_{3}$ 
and $K\left(B S_{3}\right)$ is explained here. But, for $n \geq 4$, the ring $K\left(B S_{n}\right)$ becomes devilishly complicated. The polynomials arising as minimal relations of these rings can have tremendous connections with the homotopy groups of spheres.

\section{References}

[1] R.R. Bruner, J.P.C. Greenlees, The connective K-theory of finite groups, Memoirs of the American Mathematical Society, 165, No. 785 (2003), 127, doi: $10.1090 / \mathrm{memo} / 0785$.

[2] D. Handel, On products in the cohomology of the dihedral groups, Tôhoku Math. J. (2), 45, No. 1 (1993), 13-42, doi: 10.2748/tmj/1178225952.

[3] M. Imaoka, M. Sugawara, On the K-ring of the orbit manifold $\left(S^{2 k+1} \times S^{l}\right) / D_{n}$ by the dihedral group $D_{n}$, Hiroshima Math. J., 4, No. 1 (1974), 53-70, http://projecteuclid.org/euclid.hmj/1206137151.

[4] M. Kırdar, S. Özdemir, On the K-ring of the classifying space of the generalized quaternion group, ArXiv: 1212.3087v3. 